\title{
PERMANENTLY OPTIMAL STRATEGIES IN EXTENSIVE GAMES
}

\author{
W. H. CLINGMAN
}

I. Introduction. The objective of this study is to develop a relationship between the information pattern in extensive games and the existence of permanently optimal strategies. The investigation has been restricted to zero-sum, two-person games, and the notation of Kuhn [1] is used throughout.

Morgenstern and Von Neumann [2] have demonstrated that in a two-person, zero-sum game with perfect information, each player has pure strategies which are optimal strategies. In other words, if $\mathcal{F C}_{2}\left(\tau_{1}, \tau_{2}\right)$ is the payment made to player 2 when players 1 and 2 choose strategies $\tau_{1}$ and $\tau_{2}$ respectively, there exist a $\bar{\tau}_{1}, \bar{\tau}_{2}$, and $v_{0}$ such that

$$
\begin{array}{ll}
\mathcal{H}_{2}\left(\tau_{1}, \bar{\tau}_{2}\right) \geqq v_{0} & \text { for all } \tau_{1}, \\
\mathcal{H}_{2}\left(\bar{\tau}_{1}, \tau_{2}\right) \leqq v_{0} & \text { for all } \tau_{2} .
\end{array}
$$

The strategy $\bar{\tau}_{2}$ insures player 2 that his gain will be at least $v_{0}$, independent of the strategy chosen by player 1 . Likewise, the strategy $\bar{\tau}_{1}$ insures player 1 that his loss will be no more than $v_{0}$, independent of the strategy chosen by player 2 .

Von Neumann and Morgenstern [2] also state the theorem that if both players have perfect information they have pure strategies, $\tau_{1}^{*}$ and $\tau_{2}^{*}$, which are permanently optimal.

$$
\begin{array}{ll}
\mathfrak{H}_{2}\left(\tau_{1}, \tau_{2}^{*}\right)=\max _{\tau_{2}} \mathfrak{H}_{2}\left(\tau_{1}, \tau_{2}\right) & \text { for all } \tau_{1} ; \\
\mathcal{H}_{2}\left(\tau_{1}^{*}, \tau_{2}\right)=\min _{\tau_{1}} \mathfrak{H}_{2}\left(\tau_{1}, \tau_{2}\right) & \text { for all } \tau_{2} .
\end{array}
$$

The pure strategy $\tau_{2}^{*}$ insures player 2 of maximizing his gain independent of the choice of player 1 . It follows that a permanently optimal strategy is also an optimal strategy. The converse is not true, since an optimal strategy for player 2 may not take advantage of a mistake by player 1 (i.e., player 1 not choosing an optimal strategy).

Dalkey [3] has generalized the conditions for the existence of pure, optimal strategies in terms of the information pattern of the game. He has established a necessary and sufficient condition that a twoperson game have pure, optimal strategies independent of the particular pay-off function or the probability distribution assigned to chance moves. This condition is that at any move a player knows all

Received by the editors January 27, 1965. 
preceding moves of his opponents and knows at least as much as his opponents knew when they made those moves.

In this paper the conditions are generalized for the existence of permanently optimal strategies in two-person games. It will be shown that a sufficient condition for a player to have a permanently optimal strategy (either pure or mixed) in a game $\Gamma$ is that the information withheld from the player in $\Gamma$ is inessential. That is, if the player is given this information, he will not have any new strategy which will give him a greater gain than in the original game. It will also be shown that a necessary condition for a player to have a permanently optimal strategy in a game with no chance moves is that the information withheld from the player is inessential. These results involve the payoffs and chance probabilities as well as the informational structure of the game.

II. Notations. Following the notation of Kuhn, the extensive form of a game is described as follows:

A game $\Gamma$ is a game tree $K$ with the following specifications:

1. The game tree $K$ is a finite tree with a distinguished vertex $O$ which is embedded in an oriented plane. The alternatives at a vertex $x \in K$ are the edges incident at $x$ and lying in components of $K$ which do not contain $O$ if we cut $K$ at $x$. Those vertices with alternatives are moves and those without alternatives are plays. The alternatives at each move are numbered. $A_{j}$ is defined as the set of all moves with $j$ alternatives.

2. There is a player partition of the moves into sets $B_{0}, B_{1}, B_{2}$. The moves in $B_{0}$ are called chance moves. The moves in $B_{i}$ are called personal moves of player $i$.

3. There is a partition of the moves into information sets $U$ such that each $U$ is contained in $B_{i} \cap A_{j}$ for some $i$ and $j$ and such that no $U$ contains two moves on the same unicursal path from $O$ to a particular play, $W$.

4. For each $U \subset\left(B_{0} \cap A_{j}\right), U$ is a one-element set and there exists a probability distribution, $p_{i}(U) \geqq 0, i=1,2, \cdots, j$.

5. There is a pay-off function, $h(W)$, which is an ordered pair of real numbers $\left[h_{1}(W), h_{2}(W)\right]$ for each play $W ; h_{1}(W)$ is the gain to player 1 when the result of the game is $W$.

The family of information sets contained in $B_{k}$ is denoted by $\mathfrak{u}_{k}$.

III. Proof of main results. To prove the main results for a general zero-sum, two-person game $\Gamma$, two new games, $\Gamma^{1}$ and $\Gamma^{2}$, are defined in which players 1 and 2 are given perfect information respectively. A relation is then established between strategies in $\Gamma$ and those in 
$\Gamma^{1}$ and $\Gamma^{2}$ such that related strategies result in the same final outcome. Using as a starting point the fact that in a game with perfect information both players have permanently optimal strategies, the related strategies are used to determine necessary and sufficient conditions for the existence of permanently optimal strategies in $\Gamma$. The concept of inessential information, defined in terms of $\Gamma$ and $\Gamma^{1}$ (for player 1), plays a central role in these conditions.

Given any game $\Gamma$, the game $\Gamma^{1}$ is defined in terms of the game tree and partitions for $\Gamma$ as follows:

$$
\begin{aligned}
K^{1} & =K, \\
B_{i}^{1} & =B_{i} \quad i=0,1,2, \\
U^{1} & =U \quad \text { if } U \subset B_{0} \text { or } U \subset B_{2}, \\
U^{1} & =\text { a one element set if } U^{1} \subset B_{1}, \\
p_{i}\left(U^{1}\right) & =p_{i}(U) \quad \text { if } U \subset B_{0}, \\
h_{i}^{1}(W) & =h_{i}(W) .
\end{aligned}
$$

The game $\Gamma^{2}$ is defined in an analogous fashion with respect to player 2 .

A strategy for player $k$ in $\Gamma$ is a function $\tau_{k}$ mapping $u_{k}$ into the positive integers such that $U \subset A_{j}$ implies $\tau_{k}(U) \leqq j$. The function $\tau_{0}$ maps $\mathcal{U}_{0}$ in to the positive integers and is a chance strategy. The strategy $\tau_{k}$ uniquely determines the alternative chosen by player $k$ at each move within $B_{k}$.

Let $G_{1}\left(\tau_{0}, \tau_{1}, \tau_{2}\right)=h_{1}(W)$ be the value to player 1 of the play, $W$, determined by the set of strategies, $\left(\tau_{0}, \tau_{1}, \tau_{2}\right)$.

Let $\mathfrak{F C}_{1}\left(\tau_{1}, \tau_{2}\right)$ be the expected payoff to player 1 for the pure strategies $\tau_{1}, \tau_{2}$.

$$
\mathfrak{F}_{1}\left(\tau_{1}, \tau_{2}\right)=\sum_{i} P_{i} G_{1}\left(\tau_{0}(i), \tau_{1}, \tau_{2}\right)
$$

where $\sum_{i} P_{i}=1$ and $P_{i}$ is the probability that the chance strategy will be $\tau_{0}(i)$.

We now prove the following two lemmas:

LeMMA 1. For every pair of strategies, $\tau_{1}$ and $\tau_{2}$, in $\Gamma$ there is a strategy, $\tau_{1}^{\prime}$, for player 1 in $\Gamma^{1}$ depending only on $\tau_{1}$, such that $\mathfrak{H}_{1}^{\prime}\left(\tau_{1}^{\prime}, \tau_{2}\right)$ $=\mathcal{F C}_{1}\left(\tau_{1}, \tau_{2}\right)$ and $G_{1}^{\prime}\left(\tau_{0}, \tau_{1}^{\prime}, \tau_{2}\right)=G_{1}\left(\tau_{0}, \tau_{1}, \tau_{2}\right)$.

An analogous lemma holds for $\Gamma^{2}$ with the roles of the players interchanged. 
Proof. In $\Gamma^{1}, u_{1}$ consists of one element sets and $\tau_{1}^{\prime}$ is defined over these sets as follows in terms of $\tau_{1}$ in $\Gamma$ :

$$
\tau_{\mathfrak{l}}^{\prime}(x)=\tau_{1}(U) \quad \text { where } x \in U .
$$

This definition obviously leads to the same choice of alternatives by player 1 at each move in $\Gamma^{1}$ and $\Gamma$.

Thus, the outcome, $W$, in $\Gamma$ and $\Gamma^{1}$ will be the same, and

$$
G_{1}^{\prime}\left(\tau_{0}, \tau_{1}^{\prime}, \tau_{2}\right)=G_{1}\left(\tau_{0}, \tau_{1}, \tau_{2}\right) .
$$

Since $\tau_{1}^{\prime}$ is independent of $\tau_{0}$, one also has

$$
H_{1}^{\prime}\left(\tau_{1}^{\prime}, \tau_{2}\right)=H_{1}\left(\tau_{1}, \tau_{2}\right) \text {. }
$$

Lemma 2. For any set of strategies, $\left(\tau_{0}, \tau_{1}, \tau_{2}\right)$ in $\Gamma^{1}$, there exists a strategy ${ }^{\prime} \tau_{1}$ in $\Gamma$, generally depending on $\tau_{0}$ and $\tau_{2}$, such that $G_{1}^{\prime}\left(\tau_{0}, \tau_{1}, \tau_{2}\right)$ $=G_{1}\left(\tau_{0},{ }^{\prime} \tau_{1}, \tau_{2}\right)$.

Proof. Let $W$ be the unique play determined in $\Gamma^{1}$ by the set of strategies, $\left(\tau_{0}, \tau_{1}, \tau_{2}\right)$.

' $\tau_{1}$ is defined as follows:

If $x \in U$ and $x$ is a move on the unique unicursal path from $O$ to $W$, ${ }^{\prime} \tau_{1}(U)=\tau_{1}(x)$. If $U$ contains no member of this path, ' $\tau_{1}(U)=1$. These two conditions are mutually exclusive, since the information set $U$ contains at most one member of the same play.

With this definition of ' $\tau_{1}, W$ is the unique play determined in $\Gamma$ by the set of strategies, $\left(\tau_{0},{ }^{\prime} \tau_{1}, \tau_{2}\right)$.

$$
G_{1}^{\prime}\left(\tau_{0}, \tau_{1}, \tau_{2}\right)=G_{1}\left(\tau_{0}{ }^{\prime} \tau_{1}, \tau_{2}\right) .
$$

We now precisely define the concept of inessential information, which is a central part of the main results.

DEFINITION. The information withheld from player 1 in $\Gamma$ is inessential if and only if the following is true:

If the $\tau_{1}^{*}(i)$ are the pure strategies for player 1 in $\Gamma$, there exists a $P_{1}(i) \quad\left\{P_{1}(i) \geqq 0 ; \quad \sum_{i} P_{1}(i)=1\right\}$ such that $\sum_{i} P_{1}(i) \mathcal{F C}_{1}\left[\tau_{1}^{*}(i), \tau_{2}\right]$ $\geqq \mathcal{H C}_{1}^{\prime}\left[\tau_{1}, \tau_{2}\right]$ for all $\tau_{1}$ in $\Gamma^{1}$ and for all $\tau_{2}$.

$P_{1}(i)$ defines a mixed strategy for player 1 in $\Gamma$.

We can now proceed directly to prove the following two theorems, which are the main results.

THEOREM 1. If the information withheld from player 1 in $\Gamma$ is inessential, then player 1 has at least one permanently optimal strategy in $\Gamma$.

Proof. First consider the game $\Gamma^{1}=\bar{\Gamma}$.

In the game $\bar{\Gamma}^{2}$ both players have perfect information. Thus, player 1 has a permanently optimal strategy, $\bar{\tau}_{1}$, in $\bar{\Gamma}^{2}$. 


$$
\overline{\mathfrak{F}}_{1}^{2}\left(\bar{\tau}_{1}, \tau_{2}\right) \geqq \overline{\mathfrak{F}}_{1}^{2}\left(\tau_{1}, \tau_{2}\right) \quad \text { for all } \tau_{1} \text { and } \tau_{2} \text { in } \bar{\Gamma}^{2} \text {. }
$$

Now assume that $\tau_{2}^{*}$ is a strategy for player 2 in $\bar{\Gamma}$ and that $\overline{\mathcal{F}}_{1}\left(\bar{\tau}_{1}, \tau_{2}^{*}\right)<\overline{\mathscr{H}}_{1}\left(\tau_{1}, \tau_{2}^{*}\right)$ for some $\tau_{1}$ in the game $\bar{\Gamma}$. By Lemma 1 , however, we can define the strategy $\tau_{2}^{* 2}$ in $\bar{\Gamma}^{2}$ such that $\overline{\mathcal{K}}_{1}^{2}\left(\bar{\tau}_{1}, \tau_{2}^{* 2}\right)$ $<\overline{\mathcal{F C}}_{1}^{2}\left(\tau_{1}, \tau_{2}^{* 2}\right)$. This contradicts (1).

Thus

(2) $\quad \overline{\mathscr{K}}_{1}\left(\bar{\tau}_{1}, \tau_{2}^{*}\right) \geqq \overline{\mathscr{F}}_{1}\left(\tau_{1}, \tau_{2}^{*}\right)$ for all $\tau_{1}$ and $\tau_{2}^{*}$ in the game $\bar{\Gamma}$.

Thus, player 1 has a permanently optimal strategy, $\bar{\tau}_{1}$, in the game $\Gamma^{1}=\bar{\Gamma}$.

Since the information withheld from player 1 in $\Gamma$ is inessential, there exists a $P_{1}(i)$ such that

$$
\sum_{i} P_{1}(i) \mathfrak{F}_{1}\left[\tau_{1}^{*}(i), \tau_{2}^{*}\right] \geqq \overline{\mathfrak{K}}_{1}\left[\bar{\tau}_{1}, \tau_{2}^{*}\right] \quad \text { for all } \tau_{2}^{*}
$$

Then there is an $a$ such that

$$
\mathfrak{K}_{1}\left[\tau_{1}^{*}(a), \tau_{2}^{*}\right] \geqq \overline{\mathscr{K}}_{1}\left[\bar{\tau}_{1}, \tau_{2}^{*}\right] \geqq \overline{\mathscr{H}}_{1}\left(\tau_{1}, \tau_{2}^{*}\right)
$$

for all $\tau_{2}^{*}$ and all $\tau_{1}$ in the game $\bar{\Gamma}$.

Assume that player 1 does not have a permanently optimal strategy in $\Gamma$. Then $\tau_{1}^{*}(a)$ is not permanently optimal, and there exist a $\tau_{1}^{*}(b)$ and a $\tau_{2}^{*}(c)$ such that $\mathfrak{F}_{1}\left[\tau_{1}^{*}(a), \tau_{2}^{*}(c)\right]<\mathfrak{K}_{1}\left[\tau_{1}^{*}(b), \tau_{2}^{*}(c)\right]$.

From Lemma 1 strategies $\tau_{1}^{* \prime}(a)$ and $\tau_{1}^{* \prime}(b)$ can be defined for player 1 in $\bar{\Gamma}$ such that

$$
\overline{\mathcal{F}}_{1}\left[\tau_{1}^{* \prime}(a), \tau_{2}^{*}\right] \geqq \overline{\mathscr{F}}_{1}\left(\tau_{1}, \tau_{2}^{*}\right) \quad \text { for all } \tau_{2}^{*} \text { and } \tau_{1} \text { in } \bar{\Gamma}
$$

and

$$
\overline{\mathfrak{F}}_{1}\left[\tau_{1}^{* \prime}(a), \tau_{2}^{*}(c)\right]<\overline{\mathfrak{H}}_{1}\left[\tau_{1}^{* \prime}(b), \tau_{2}^{*}(c)\right] .
$$

(4) and (5) are contradictory, proving the theorem.

Q.E.D.

TheOREM 2. If the game $\Gamma$ contains no chance moves and if player 1 has a permanently optimal strategy in $\Gamma$, then the information witheld from player 1 is inessential.

Proof. Let $\tau_{1}^{0}$ be a permanently optimal strategy for player 1 in $\Gamma$.

Now assume that the information withheld from player 1 in $\Gamma$ is essential. Then there exists a $\tau_{1}(a)$ in $\Gamma^{1}$ and a $\tau_{2}(b)$ such that $\mathfrak{F}_{1}\left[\tau_{1}^{0}, \tau_{2}(b)\right]<\mathcal{F C}_{1}^{\prime}\left[\tau_{1}(a), \tau_{2}(b)\right]$.

Since the game contains no chance moves, Lemma 2 is valid for the 
functions $\mathcal{H C}_{1}^{\prime}$ and $\mathcal{H}_{1}$. A strategy ' $\tau_{1}(a)$ in $\Gamma$ can be defined such that: $\mathfrak{F C}_{1}\left[{ }^{\prime} \tau_{1}, \tau_{2}(b)\right]=\mathcal{H C}_{1}^{\prime}\left[\tau_{1}(a), \tau_{2}(b)\right]$. Thus, $\mathfrak{F C}_{1}\left[\tau_{1}^{0}, \tau_{2}(b)\right]<\mathfrak{F}_{1}\left[{ }^{\prime} \tau_{1}, \tau_{2}(b)\right]$.

This last inequality contradicts the premise that $\tau_{1}^{0}$ is a permanently optimal strategy, thus proving the theorem.

Q.E.D.

\section{BIBLIOGRAPHY}

1. H. W. Kuhn, Contributions to the theory of games, Vol. 2, Princeton Univ. Press, Princeton, N. J., 1953; pp. 193-216.

2. J. von Neumann and O. Morgenstern, Theory of games and economic behavior, Princeton Univ. Press, Princeton, N. J., 1947.

3. N. Dalkey, Contributions to the theory of games, Vol. 2, Princeton Univ. Press, Princeton, N. J., 1953; pp. 217-243.

Texas Instruments Incorporated, Dallas, Texas 